\title{
Revisited: Haemodynamic Instability and Endocrine Response During Endotracheal Tube-Placement. A Prospective, Randomized Trial Using Topical Lidocaine and a Lightwand
}

\author{
B. Dünges ${ }^{1}$, F. Heid ${ }^{1}$, M. Dauster ${ }^{1}$, U. Strecker ${ }^{1}$, B. Röhrig ${ }^{3}$ and A.M. Brambrink ${ }^{*}, 1,2$ \\ ${ }^{1}$ Department of Anesthesiology, Johannes Gutenberg-University Mainz, Germany \\ ${ }^{2}$ Department for Anesthesiology and Peri-Operative Medicine, Oregon Health \& Science University, Portland, OR \\ 97239, USA \\ ${ }^{3}$ Institute of Medical Biostatistics, Epidemiology and Informatics (IMBEI), Johannes Gutenberg-University, Germany
}

\begin{abstract}
Endotracheal intubation via direct laryngoscopy frequently provokes cardiovascular side-effects. Although using a lightwand intubation device reduces laryngeal stimulation, previous reports indicated a similar stress response compared to classical laryngoscopy. We hypothesized that endotracheal tube (ET) placement itself elicits haemodynamic instability and that topical anaesthesia can attenuate this response.

Methods: 30 patients were randomized to three groups $(\mathrm{n}=10$ each). After induction of general anaesthesia (fentanyl, etomidate, vecuronium) $5 \mathrm{ml}$ of test solution was applied to laryngo-tracheal structures via a lightwand guided EDGARTube $^{\circledR}$. Control group received $5 \mathrm{ml}$ saline $0.9 \%$, group lido $1 \% 5 \mathrm{ml}$ lidocaine $1 \%$, and group lido $2 \% 5 \mathrm{ml}$ lidocaine $2 \%$. After 2 minutes of bag-mask ventilation lightwand guided ET placement was performed. Invasive systolic arterial pressure (SAP, $\mathrm{mmHg})$, heart rate $(\mathrm{HR}, \mathrm{bpm})$ and arterial plasma concentrations of catecholamines ([adr][nor], pcg ml-) were determined.

Results: After ET placement control group patients showed increased HR and SAP (mean $\Delta \mathrm{HR}=15.3$; mean $\Delta \mathrm{SAP}=$ 45.6) compared to both lido groups ( $\Delta$ HR: lido1\%/2\% $=5.8 / 3.7 ; \Delta$ SAP: lido1\%2\% $=8.7 / 13.0$ ). Catecholamine concentrations also increased only in the control group (mean $\Delta[\mathrm{adr}]=101.43 ; \Delta[$ nor] $=89.41)$ but not in lido groups $(\Delta$ [adr]: lido $1 \% / 2 \%=-12.93 / 7.05 ; \Delta$ [nor]: lido $1 \% / 2 \%=-6.61 /-30.55)$. Effect size calculation indicated strong clinical effects of topical lidocaine for almost all variables $(\mathrm{ES}>0.8)$.

Conclusion: ET placement into the non-anaesthesized trachea causes haemodynamic and endocrine stress even if direct laryngoscopy is omitted. Topical anaesthesia effectively reduces this response.
\end{abstract}

Keywords: Airway, stimulation, anaesthesia techniques, topical, laryngoscopy.

\section{INTRODUCTION}

Direct laryngoscopy for endotracheal intubation is frequently associated with an acute endocrine and cardiovascular stress-response that might be harmful to patients who are at risk for cardiovascular or intracranial complications [1]. Previous research determined that direct laryngoscopy can cause an increase of blood pressure and heart rate by stimulating pharyngeal and laryngeal proprioceptors [2]. However, even if laryngoscopy is omitted during endotracheal tube placement haemodynamic alteration has been observed [3]. In contrast to direct-vision laryngoscopy, indirect lightwand guided intubation using commercially available devices like, e.g. Trachlight ${ }^{\circledR}$ (Laerdal Medical AS, Stavanger, Norway), does not require gross manipulation of laryngeal structures. A powerful light-source at the distal end of the lightwand is used for indirect guidance of the tip of the breathing tube into the trachea without direct visualization of the glottic opening. When in-line with the tip of the endotracheal tube

*Address correspondence to this author at the Department of Anesthesiology, Johannes Gutenberg-University Mainz, Germany;

E-mail: brambrin@ohsu.edu
(ET), the light source creates a profound transcutaneous glow which can initially be observed at the level of the cricoid membrane (light shines into the glottic opening; the tip of the ET is in immediate proximity of the vocal cords), and subsequently at the jugular notch when the ET is advanced into the upper trachea. However, even when direct laryngoscopy is avoided using a lightwand, cardiovascular stress responses are seen to occur during endotracheal intubation [4-7]. This corresponds well with our anecdotal experience where gentle handling of the laryngoscope is usually not associated with haemodynamic fluctuation, whereas the insertion of the ET is frequently followed by tachycardia and hypertension. Indeed, it has been postulated that the circulatory response after tracheal intubation could in fact be due to the ET placement and cuff inflation itself rather than the stimulation by direct laryngoscopy [3].

Previous studies have already used lidocaine as a topical anaesthetic prior to endotracheal intubation to investigate the effects on cardiovascular and endocrine parameters. However they did not discriminate between the suppression of the stress response due to oropharyngeal as opposed to endotracheal stimulation [8-11]. 
Therefore, the aim of our double-blinded and randomized investigation was to clarify the impact of endotracheal placement of an ET on the stress response associated with airway management after induction of general anaesthesia. We hypothesized that tracheal intubation - even if direct laryngoscopy is omitted - causes an adverse change in haemodynamic variables along with a rise in serum catecholamine levels, and that topical anaesthesia of the larynx and the upper trachea prior to intubation attenuates this response.

\section{PATIENTS AND METHODS}

With approval of the local Ethics Committee and written informed consent, 30 patients (ASA physical status III) scheduled for elective orthopaedic or abdominal surgery under general anaesthesia were enrolled in our study. The investigation was conducted according to the standards of good clinical practice and the Helsinki Declaration. The following exclusion criteria were applied: known difficult airway, history of surgery in the head-throat-area, lidocaine intolerance, multiple allergies, and necessity of a gastric tube.

Patients were assigned to one of the three following groups $(\mathrm{n}=10$ each) by a computer-generated randomization list with the designated topical treatment in brackets: control group (5 ml saline $0.9 \%$ ), group lido $1 \%$ (5 ml lidocaine 1\%) and group lido $2 \%$ (5 ml lidocaine $2 \%)$. All test solutions were prepared by an independent person and blinded for all individuals involved in the study. In combination with a light-wand device a special endotracheal breathing tube (Edgar $^{\circledR}$ tube, endotracheal drug and gas application during resuscitation, Rüsch, Kernen, Germany) was used to spray the test solutions directly onto the glottic opening, which allowed them to spread across vocal cords and upper parts of the trachea. In principal, the Edgar ${ }^{\circledR}$ tube is based on a conventional endotracheal tube with an additional instillation line integrated within the tube wall, which enables precise drug instillation or gas monitoring [12]. Patients received 10 $\mathrm{mg}$ of oxazepam p.o. $90 \mathrm{~min}$. prior to induction of GA and if prescribed - their regular cardiovascular medication (betablockers, $\mathrm{Ca}^{++}$- antagonists, nitrates). Standard monitoring according to the American Society of Anesthesiologists (ASA) was applied and a radial artery was cannulated for continuous intravascular blood pressure monitoring after a negative Allen's test. We exclusively studied ASA III patients because their cardiovascular system frequently is more unstable than in healthy patients, yet cardiovascular and endocrine effects may emerge more pronounced. Additionally, our institutional standard operating procedures determine that these patients are monitored by an arterial line if major surgery is carried out. Facing its potential risks, an arterial line may not be a suitable monitoring for an ASA I or II patient. All physiological variables were monitored continuously, and arterial blood pressures and heart rate were electronically documented throughout the experimental period for subsequent analysis.

The arterial line was also used for arterial blood samples to analyze for catecholamine and lidocaine plasma concentrations. Intravenous induction of anaesthesia was standardized (fentanyl $0.003 \mathrm{mg} \mathrm{kg}^{-1}$, etomidate, $0.2 \mathrm{mg} \mathrm{kg}^{-1}$ and vecuronium $0.1 \mathrm{mg} \mathrm{kg}^{-1}$ ). Ninety seconds after intravenous induction the Edgar ${ }^{\circledR}$ tube was positioned directly above the glottis using a commercially available lightwand (Trachlight ${ }^{\circledR}$ ), thus omitting direct laryngoscopy. The test solution $(5 \mathrm{ml})$ was applied. ET and lightwand were removed temporarily and kept in a sterile container. The patients were than bag-mask ventilated for an additional two minutes $\left(\mathrm{FiO}_{2}=\right.$ $1.0)$, followed by lightwand-guided intubation of the trachea. After confirmation of correct ET placement (end-tidal carbon dioxide tension, bilateral chest auscultation) anaesthesia then was maintained employing isoflurane (endtidal Vol.\% 0.91.1). The airway related procedures in all patients were performed by the same independent senior anaesthesiologist, who has long-term experience with both the Trachlight ${ }^{\mathbb{B}}$ as well as the Edgar ${ }^{\circledR}$ tube. The study protocol dictated the abortion of lightwand guided intubation following two unsuccessful attempts or $>30$ seconds, whatever came first.

Arterial blood samples were obtained at five time points: following induction of anaesthesia (baseline); following application of the test solution (drug); immediately after endotracheal tube placement and cuff-inflation (ETP); and five minutes $(5 \mathrm{~min})$ and ten minutes $(10 \mathrm{~min})$ after endotracheal tube placement. Blood samples were heparinized (15 I.U. heparin $\mathrm{ml}^{-1}$ ), cooled to $4^{\circ} \mathrm{C}$ and centrifuged (4000 rpm, 5 $\mathrm{min})$ within $30 \mathrm{~min}$. Plasma samples were then stored at $70^{\circ} \mathrm{C}$ until en-block analysis.

Plasma catecholamines (adrenaline, noradrenaline) were extracted and concentrated with a commercial extraction kit (Recipe, Munich, Germany) and then analyzed by ion pair high-performance liquid chromatography (HPLC 1050, Hewlett-Packard, Palo Alto, CA, USA) with electrochemical detection (HP 1049 A, Hewlett-Packard). Measurement of lidocaine plasma concentration was performed using a UV detector (HP MWD 1050 Hewlett-Packard). For both analyzes a LiChrospher 60 RP-select B HPLC-column was used (5 $\mu \mathrm{m}, 125$ mm x 4 mm, Merck, Darmstadt, Germany).

\section{STATISTICAL ANALYSIS}

Statistical analysis was performed using SigmaStat ${ }^{\circledR}$ for windows, version 3.1 (Systat Software, Inc., San Jose, CA, USA).

Statistical measurements were calculated for the determined characteristics. In addition to absolute values of each variable, we calculated the difference $\Delta$ (=delta-values) for every single patient $(\Delta$ : difference between drug application and ETP).

To test the statistical significance of the delta-values between control group and lido $1 \%$, between control group and lido $2 \%$ as well as between lido $1 \%$ and lido $2 \%$, Student's ttest was performed. Statistical analyses were done in an explorative manner. The outcome of a statistical test with a pvalue $<.05$ was called significant. To obtain clinically more relevant information we additionally calculated the effectsize as described by Cohen [13]. Values of 0.2 indicate small clinical effects, whereas values of 0.5 indicate medium and values exceeding 0.8 strong effects, respectively.

\section{RESULTS}

There was no difference in patient demographics between the groups (Table 1). One male patient, initially randomized to group lido $2 \%$, was excluded from the study because trachlight-guided intubation failed and the ET was placed via 
conventional laryngoscopy. There was no adverse reaction (e.g. coughing, laryngo- or bronchospasm) noted in any of the patients following topical application of the test solutions.

Table 1. Demographic Data. Values are Mean \pm SD or Number of Patients. No Significant Differences were Noted $(p>0.05)$

\begin{tabular}{|c|c|c|c|}
\hline & $\begin{array}{c}\text { Group Lido } \\
\mathbf{2 \%}\end{array}$ & $\begin{array}{c}\text { Group Lido } \\
\mathbf{1 \%}\end{array}$ & Control Group \\
\hline \hline & $(\mathrm{n}=9)$ & $(\mathrm{n}=10)$ & $(\mathrm{n}=10)$ \\
\hline Sex $(\mathrm{m} / \mathrm{f})$ & $6 / 3$ & $7 / 3$ & $5 / 5$ \\
\hline Age $(\mathrm{yr})$ & $60.3 \pm 13.6$ & $55.6 \pm 12.3$ & $53.2 \pm 10.5$ \\
\hline Weight $(\mathrm{kg})$ & $83 \pm 10.9$ & $81.1 \pm 16.4$ & $76.3 \pm 18.4$ \\
\hline
\end{tabular}

Fig. (1a-d) summarizes the changes in haemodynamic variables during endotracheal tube placement (cardiovascular stress response) for all timepoints. At baseline as well as after topical drug application both heart rate (HR; Fig. 1a) and systolic arterial pressure (SAP; Fig. 1c) were similar between groups. Immediately following ET placement HR and SAP increased in patients who had received topical saline (mean $\triangle \mathrm{HR}=15.3 \mathrm{bpm}$; standard deviation $(\mathrm{sd})=9.0$; mean $\Delta \mathrm{SAP}=45.6 \mathrm{mmHg} ; \mathrm{sd}=18.8)$ (Table $\mathbf{2 a}, \mathbf{b}$; Fig. $\mathbf{1 b}$ and $1 \mathbf{d}$, respectively). In contrast, patients who had received topical lidocaine showed only minor changes in haemodynamic variables, irrespective of the lidocaine concentration applied (mean $\Delta \mathrm{HR}$ : lido $1 \%=5.8 \mathrm{bpm}$; sd $=8.9$; lido $2 \%=$ $3.7 \mathrm{bpm}$; $\mathrm{sd}=15.9$; mean $\Delta \mathrm{SAP}$ : lido $1 \%=8.7 \mathrm{mmHg}$; $\mathrm{sd}=$ 18.9; lido $2 \%=13.0 \mathrm{mmHg}$; sd = 16.7). Effect size calculation indicated a strong effect of topical lidocaine on both variables (ES control vs lido groups $>1$; Table 2a,b).

Fig. (2a-d) illustrates the changes in catecholamine plasma levels following endotracheal tube placement (endocrine stress response). Baseline levels of noradrenaline ([nor]; Fig. 2c) were similar, while unexpectedly adrenaline levels [adr] in group lido $2 \%$ were lower than in the other
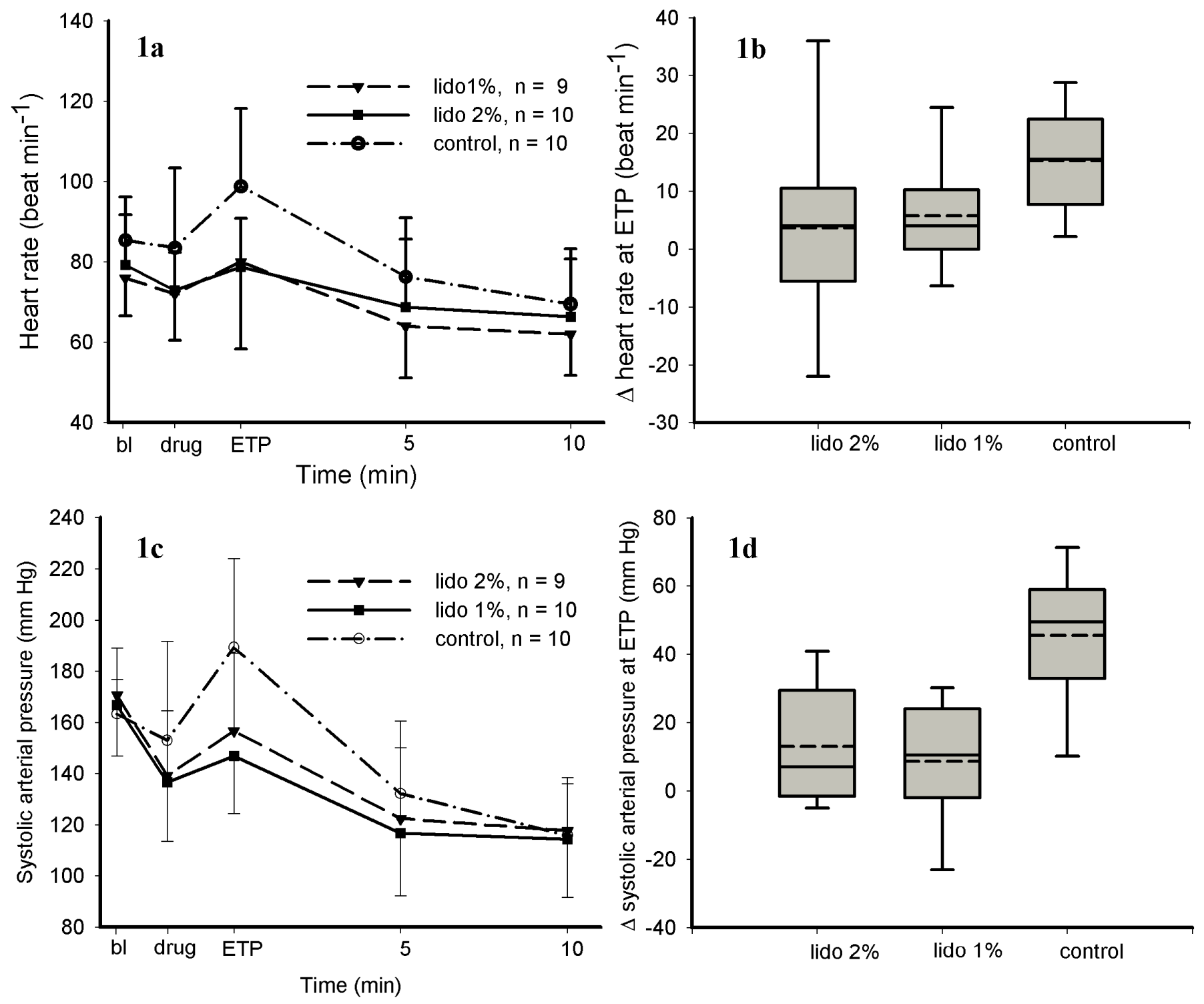

Fig. (1a-d). Cardiovascular stress response. 
Table 2a-d. Delta-Values for All Variables, Displaying the Effects of Endotracheal Tube Placement and Cuff-Inflation. Descriptive Statistics, Differences Between Groups are Indicated by p-Value and Effect Size (13) (Delta-Values = Change Between Drug Application and Endotracheal Tube Placement; Med = Median; CIM = 95\% Confidence Interval of Mean)

a. $\Delta$ Heart Rate (Beats Min $^{-1}$ )

\begin{tabular}{|c|c|c|c|c|c|c|c|c|}
\hline Group & Mean & Std Dev & Min & Max & Med & CIM & $\mathbf{2 5 \%}$ & $\mathbf{7 5 \%}$ \\
\hline \hline control & 15.3 & 9.0 & 2 & 29 & 15.5 & 6.46 & 9.0 & 21.0 \\
\hline $1 \%$ & 5.8 & 8.9 & -7 & 26 & 4.0 & 6.42 & 0.0 & 10.0 \\
\hline $2 \%$ & 3.7 & 15.9 & -22 & 36 & 4.0 & 12.23 & -3.3 & 9.3 \\
\hline
\end{tabular}

p-value $1 \%$ vs $2 \%=0.720 ;$ p control $v s 1 \%=0.030 ;$ p control $v s 2 \%=0.063$; ES control $v s 1 \%=1.05$; ES control $v s 2 \%=1.29$.

b. $\Delta$ Systolic Arterial Pressure (mmHg)

\begin{tabular}{|c|c|c|c|c|c|c|c|c|}
\hline Group & Mean & Std Dev & Min & Max & Med & CIM & $\mathbf{2 5 \%}$ & $\mathbf{7 5 \%}$ \\
\hline \hline control & 45.6 & 18.8 & 8 & 72 & 49.5 & 13.43 & 34.0 & 57.0 \\
\hline $1 \%$ & 8.7 & 18.9 & -23 & 31 & 10.5 & 13.52 & 5.0 & 24.0 \\
\hline $2 \%$ & 13.0 & 16.7 & -5 & 41 & 7.0 & 12.86 & 0.3 & 27.8 \\
\hline
\end{tabular}

p $1 \%$ vs $2 \%=0.608 ;$ p control $v s 1 \%=<0.001 ;$ p control $v s 2 \%=<0.001$; ES control $v s 1 \%=1.97$; ES control $v s 2 \%=1.74$.

c. $\Delta$ Arterial Plasma Concentration of Adrenaline (pcg ml ${ }^{-1}$ )

\begin{tabular}{|c|c|c|c|c|c|c|c|c|}
\hline Group & Mean & Std Dev & Min & Max & Med & CIM & $\mathbf{2 5 \%}$ & $\mathbf{7 5 \%}$ \\
\hline \hline control & 101.43 & 127.67 & 14.81 & 399.62 & 41.3 & 90.59 & 19.1 & 178.1 \\
\hline $1 \%$ & -12.93 & 33.86 & -62.27 & 60.81 & -10.5 & 23.95 & -17.9 & -7.2 \\
\hline $2 \%$ & 7.05 & 28.12 & -33.22 & 67.0 & 0.3 & 21.54 & -3.3 & 11.7 \\
\hline
\end{tabular}

p $1 \%$ vs $2 \%=0.182$; p control vs $1 \%=0.014$; p control vs $2 \%=0.045$; ES control $v s 1 \%=0.9$; es control $v s 2 \%=0.74$.

d. $\Delta$ Arterial Plasma Concentration of Noradrenaline (pcg ml $\left.{ }^{-1}\right)$

\begin{tabular}{|c|c|c|c|c|c|c|c|c|}
\hline Group & Mean & Std Dev & Min & Max & Med & CIM & $\mathbf{2 5 \%}$ & $\mathbf{7 5 \%}$ \\
\hline \hline control & 89.41 & 90.95 & 7.65 & 258.42 & 52.9 & 69.91 & 28.8 & 139.7 \\
\hline $1 \%$ & -6.61 & 43.68 & -55.69 & 101.20 & -18.3 & 31.25 & -29.7 & 11.9 \\
\hline $2 \%$ & -30.55 & 64.15 & -136.83 & 30.78 & -13.5 & 49.31 & -79.4 & 21.9 \\
\hline
\end{tabular}

p $1 \%$ vs $2 \%=0.351 ;$ p control $v s 1 \%=0.008$; p control $v s 2 \%=0.005 ;$ ES control $v s 1 \%=1.06$; ES control $v s 2 \%=1.32$.

experimental groups. Parallel to the changes observed in haemodynamic variables, [adr] and [nor] increased in response to endotracheal intubation in patients which received only topical saline (mean $\Delta[\mathrm{adr}]=101.43 \mathrm{pcg} \mathrm{ml}^{-1}$; $\mathrm{sd}=$ 127.67; mean $\Delta$ [nor $]=89.41 \mathrm{pcg} \mathrm{ml}^{-1} ; \mathrm{sd}=90.95$ ) (Table $\mathbf{2 c , d}$; Fig. 2a and $\mathbf{2 b}$, respectively). In contrast, with topical lidocaine and independent of the respective concentration used, catecholamine concentrations remained unaltered or even slightly decreased following ET placement. (mean $\Delta$ [adr]: lido $1 \%=-12.93 \mathrm{pcg} \mathrm{ml}^{-1} ; \mathrm{sd}=33.86$; lido $2 \%=7.05$ pcg $\mathrm{ml}^{-1} ; \mathrm{sd}=28.12 ;$ mean $\Delta$ [nor]: lido $1 \%=-6.61 \mathrm{pcg} \mathrm{ml}^{-1}$; $\mathrm{sd}=43.68$; lido $\left.2 \%=-30.55 \mathrm{pcg} \mathrm{ml}^{-1} ; \mathrm{sd}=64.15\right)$. Effect size calculation indicated a strong clinical effect of topical lidocaine on catecholamine release (ES $>0.8$; except mean $\Delta$ [adr] control $v s$ lido 2\%: ES = 0.79) (Table 2a-d) (complete data with means and standard deviations displayed in appendix section a-d). Table 3 displays the time course of changes in plasma lidocaine concentrations for the three experimental groups. All patients had a small baseline lidocaine plasma contamination as a result from routine flushing of the arterial line with a lidocaine containing solution $(0.2 \mathrm{mg}$ lidocaine $\mathrm{ml}$ saline $\left.^{-1}\right)$ as it is part of the standard operating procedures at the study centre. Early after topical application, lidocaine plasma levels increased significantly compared to baseline and the control group (topical saline). The maximum observed plasma concentration of lidocaine was $2.4 \mu \mathrm{g} \mathrm{ml}^{-1}$ in a patient after $2 \%$ lidocaine. There were no differences in mean lidocaine plasma levels between the two lidocaine treatment groups.

\section{DISCUSSION}

This study resulted in four important findings: 1. placement of an ET into the non-anesthetized trachea following intravenous induction of GA results in significant haemodynamic response with increased HR and SBP accompanied by 

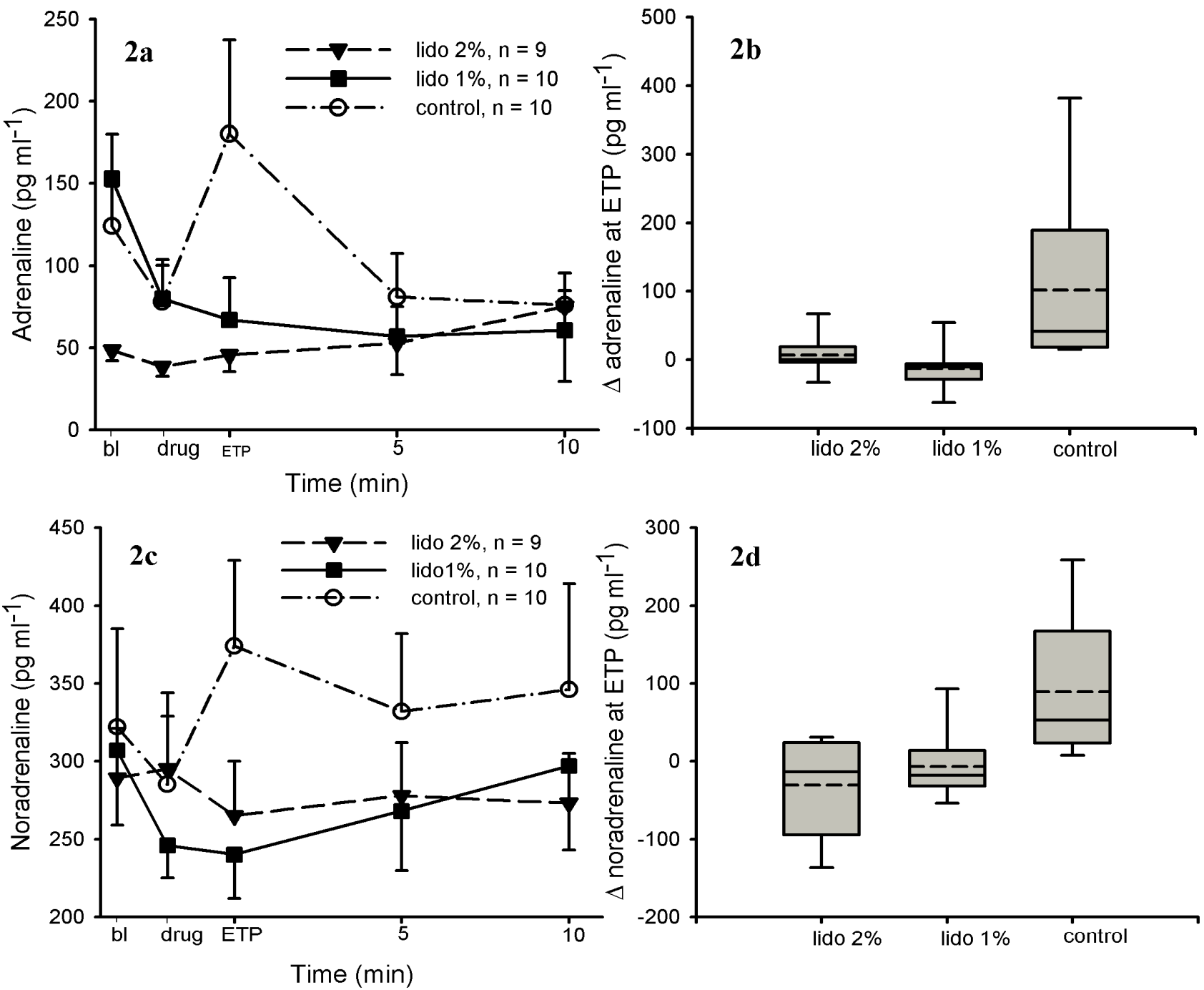

Fig. (2a-d). Endocrine stress response.

a parallel rise of plasma catecholamine levels, i.e. adrenaline and noradrenaline; 2 . this stress response occurs even if conventional laryngoscopy is omitted and an indirect technique, i.e. lightwand-guided endotracheal intubation, is used; 3 . topical anaesthesia using $1 \%$ lidocaine $(5 \mathrm{ml}, 50 \mathrm{mg})$ of larynx and upper trachea two minutes prior to endotracheal tube placement blunts both, the cardiovascular and the endocrine stress-responses observed without topical anaesthesia; 4. higher lidocaine concentrations $(2 \% ; 100 \mathrm{mg})$ render no additional benefits, but do not result in toxic plasma levels.

Table 3. Changes in Lidocaine Arterial Plasma Concentration $\left(\mu \mathrm{g} \mathrm{ml}^{-1}\right)$ During the Observational Period. Data are Displayed as Mean \pm SD

\begin{tabular}{|c|c|c|c|}
\hline & Group Lido 2\% & Group Lido 1\% & Control Group \\
\cline { 2 - 4 } & $\mathbf{( n = 9 )}$ & $\mathbf{( n = 1 0 )}$ & $\mathbf{( n = 1 0 )}$ \\
\hline \hline baseline & $0.37 \pm 0.25$ & $0.48 \pm 0.34$ & $0.43 \pm 0.25$ \\
\hline drug & $1.37 \pm 0.41$ & $1.11 \pm 0.39$ & $0.69 \pm 0.25$ \\
\hline ETP & $1.19 \pm 0.52$ & $1.12 \pm 0.44$ & $0.58 \pm 0.30$ \\
\hline $5 \mathrm{~min}$ & $1.20 \pm 0.57$ & $1.29 \pm 0.35$ & $0.61 \pm 0.18$ \\
\hline 10min & $1.24 \pm 0.25$ & $1.18 \pm 0.41$ & $0.72 \pm 0.42$ \\
\hline
\end{tabular}

Except baseline for all timepoints control group $v s$ lidocaine groups $\mathrm{p}<0.05$.
Since their introduction, lightwand-guided endotracheal intubation using devices like the Trachlight ${ }^{\circledR}$ have been considered a more gentle and less invasive technique than direct laryngoscopy. However, the circulatory stress response with Trachlight ${ }^{\circledR}$-assisted endotracheal intubation was beneficial only in ASA I but not in ASA II patients when compared to conventional direct laryngoscopy [4]. Moreover, subsequent investigations were unable to detect any differences in circulatory responses between traditional laryngoscopy- and lightwand-guided intubation of the trachea [5-7]. It was no surprise that some authors concluded that the avoidance of direct laryngoscopy using a lightwand may in fact play only a minor role in decreasing the haemodynamic stress response [3]. They speculated that the endotracheal placement of the breathing tube represents the more relevant stimulus during the procedure. Our study design was specifically tailored to further clarify this issue: 1. the use of a lightwand (Trachlight ${ }^{\circledR}$ ) allowed the avoidance of any stress response caused by conventional laryngoscopy and allowed us to observe only changes caused by placement of the ET. Here, it is important to clarify, that our work in principal did not depend on the use of a lightwand - which appears somehow complex. Our intention was to isolate the impact of the endotracheal stimulus which is caused by tube insertion and 
cuff-insufflation. Thus, also an oral fibre-optic approach would have been an appropriate technique to achieve this aim. 2. By spraying topical anaesthesia to larynx and upper trachea we were able to attenuate this effect and 3. the use of two different concentrations of lidocaine enabled us to identify a clinical relevant dose/response relationship. We decided to exclusively study patients of ASA physical status III, because their perioperative monitoring routinely included arterial blood pressure measurements when patients are scheduled for moderate to high risk surgery at our institution.

Analysis of our data confirmed the hypothesis that ET placement, independently of direct laryngoscopy, presents a significant stimulus that results in potentially relevant haemodynamic alterations. Patients who received normal saline (control group) after induction of general anesthesia had a pronounced haemodynamic reaction (HR and SBP) following lightwand-guided ET placement, which was accompanied by an increase in adrenaline and noradrenaline plasma levels.

Some of our results are in accordance, and others are in disagreement with previous findings: one report describes a cardiovascular stress-response immediately after Trachlight ${ }^{\mathbb{B}}$ guided endotracheal intubation but increases in blood catecholamine levels were absent at the same time points [7]. Another investigation observed a slight but insignificant increase of adrenaline and noradrenaline concentration in ASA I patients two and five minutes after laryngoscopic endotracheal intubation and induction of general anaesthesia, which occurred in conjunction with increases in HR and SAP [14]. The absence or non-significance of a catecholamine response to endotracheal intubation in previous studies may be explained by the fact that catecholamine levels of conscious patients were referenced as "baseline". In contrast, in our study "baseline" was defined right after induction of anaesthesia. Application of intravenous hypnotics and opioids in the attempt to induce general anaesthesia is generally accompanied by a decrease in plasma catecholamine levels [15], and this initial decrease may have masked a relative increase in catecholamine levels following ET placement in the other studies cited [7]. It is important to note that endogenous catecholamines have a relatively short plasma half life of one to three minutes [16]. This suggests that blood samples obtained earlier than two minutes after ET placement may have detected even more pronounced rises in response to tracheal stimulation [14].

Though a variety of anaesthetic techniques and drugs are available to control the haemodynamic response to endotracheal intubation [17], topical lidocaine seems an attractive alternative. In contrast to, e.g. intravenous beta blockers or high doses of opioids, negative effects on cardiovascular stability seem rather unlikely with topical lidocaine [18]. Long-term clinical experience suggests that attenuation of laryngotracheal stimuli using topical lidocaine is a safe technique. For example the Laryngo Tracheal Anaesthesia kit
$\left(\mathrm{LTA}^{\circledR}\right.$ kit, $10 \mathrm{ml}$ of $4 \%$ lidocaine, Abbott Laboratories, North Chicago, IL, USA) has been successfully used for topical laryngotracheal anaesthesia for about 30 years [19]. However, proper use of the LTA kit requires direct visualization of the glottic structures and therefore was not integrable in our study design. We chose the Edgar tube to provide topical lidocaine to the glottis in conjunction with a lightwand to avoid direct laryngoscopy. This approach could be a reasonable technique for endotracheal tube placement in patients at increased risk for cardiovascular deterioration who require an operative procedure under general anaesthesia with an endotracheal tube. An alternative and more sophisticated route of lidocaine application like ultrasound nebulization [20] could be employed to anesthetize the airway prior to endotracheal intubation, although this would take more time.

Both lidocaine concentration tested (1\% and $2 \%)$ effectively blunted the ET placement related stress response and were not accompanied by adverse effects. The maximum observed plasma concentration of lidocaine was $2.4 \mu \mathrm{g} \mathrm{ml}^{-1}$, which is far below the toxic threshold of $6.0 \mu \mathrm{g} \mathrm{ml}^{-1}$ reported in literature [21]. In fact, topical application of lidocaine appears to be safe even at much higher dosages than used in our study. When topical lidocaine was provided to healthy volunteers during fiberoptic intubation at an average dose of $8.8 \mathrm{mg} \mathrm{kg}^{-1}$ (roughly 8 -times more than in this study) the lidocaine plasma levels did not exceed values above $5 \mu \mathrm{g} / \mathrm{ml}^{-1}$, [20], i.e. remained within the currently accepted safety margin [21].

Our findings may be limited by the fact, that we did not monitor depth of anaesthesia e.g. by means of processed electroencephalography (BIS). Doses that are entirely based on $\mathrm{mg} \mathrm{kg}^{-1}$ probably produce different depths of anaesthesia in a given population - which may have affected our results. Additionally, the authors state, that a more profound induction of anesthesia before tracheal tube insertion may also have influenced the results of this study. However, if randomisation may outweigh these limitations is questionable.

In conclusion, this study demonstrated that the placement of an ET into the non-anesthetized trachea of patients under general anaesthesia causes a significant haemodynamic and endocrine stress response, regardless of the omission of conventional laryngoscopy. We have shown that this response was avoided by lightwand guided intubation in combination with the use of topical anesthesia of the laryngotracheal area prior to ET placement. This represents an alternative approach to airway management in patients at risk of cardiovascular or cerebrovascular complications.

\section{SUPPLEMENTARY MATERIAL}

Complete data (descriptive statistics) of all variables and all time-points and diagrams of the technical equipment are displayed in appendix a $-\mathrm{d}$ (data), appendix e (Edgar ${ }^{\mathbb{Q}}-$ tube and $\mathrm{f}\left(\right.$ Trachlight $\left.^{\mathbb{B}}\right)$. 


\section{Appendix a-d}

Descriptive statistics for all variables and all time-points (Med=median; 25\%/75\% = percentiles).

a. Heartrate (beats $\min ^{-1}$ )

baseline

\begin{tabular}{lccccccc} 
Group & Mean & Std Dev & Min & Max & Med & $\mathbf{2 5 \%}$ & $\mathbf{7 5 \%}$ \\
\hline control & 85.4 & 11.4 & 64 & 99 & 87.5 & 79 & 94 \\
$1 \%$ & 79.2 & 13.2 & 63 & 105 & 77.5 & 68 & 87 \\
$2 \%$ & 75.9 & 10.4 & 59 & 93 & 78 & 71.5 & 80 \\
\hline
\end{tabular}

drug

\begin{tabular}{lccccccl} 
Group & Mean & Std Dev & Min & Max & Med & $\mathbf{2 5 \%}$ & $\mathbf{7 5 \%}$ \\
\hline control & 83.5 & 21.0 & 66 & 127 & 75.5 & 67.0 & 100 \\
$1 \%$ & 72.9 & 10.0 & 52 & 84 & 76.5 & 64.0 & 80 \\
$2 \%$ & 71.9 & 12.6 & 50 & 80 & 76.0 & 63.3 & 79.5 \\
\hline
\end{tabular}

etp

\begin{tabular}{lccccccl} 
Group & Mean & Std Dev & Min & Max & Med & $\mathbf{2 5 \%}$ & $\mathbf{7 5 \%}$ \\
\hline control & 98.8 & 20.5 & 60 & 129 & 98.5 & 82 & 109 \\
$1 \%$ & 78.7 & 12.7 & 52 & 95 & 78 & 73 & 90 \\
$\mathbf{2} \%$ & 75.6 & 19.2 & 54 & 115 & 71 & 62.7 & 83.7 \\
\hline
\end{tabular}

\section{5 min}

\begin{tabular}{lccccccl} 
Group & Mean & Std Dev & Min & Max & Med & $\mathbf{2 5 \%}$ & $\mathbf{7 5 \%}$ \\
\hline control & 75.3 & 17.0 & 49 & 102 & 76 & 62 & 91 \\
$1 \%$ & 68.7 & 17.9 & 47 & 101 & 64.5 & 57 & 77 \\
$2 \%$ & 62.7 & 13.9 & 45 & 83 & 66 & 48 & 70.7 \\
\hline
\end{tabular}

10 min

\begin{tabular}{lccccccc} 
Group & Mean & Std Dev & Min & Max & Med & $\mathbf{2 5 \%}$ & $\mathbf{7 5 \%}$ \\
\hline control & 69.5 & 14.5 & 50 & 97 & 70.5 & 57 & 78 \\
$1 \%$ & 66.3 & 15.3 & 47 & 101 & 67 & 57 & 71 \\
$2 \%$ & 61.2 & 10.8 & 45 & 80 & 62 & 51 & 67 \\
\hline
\end{tabular}

b. Systolic arterial pressure (mmHg).

baseline

\begin{tabular}{lccccccc} 
Group & Mean & Std Dev & Min & Max & Med & $\mathbf{2 5 \%}$ & $\mathbf{7 5 \%}$ \\
\hline control & 163.2 & 14.19 & 144 & 186 & 163 & 148 & 173 \\
$1 \%$ & 166.7 & 20.95 & 126 & 193 & 170 & 153 & 181 \\
$2 \%$ & 170.2 & 20.44 & 131 & 198 & 172 & 159 & 185 \\
\hline
\end{tabular}

drug

\begin{tabular}{lccccccc} 
Group & Mean & Std Dev & Min & Max & Med & $\mathbf{2 5 \%}$ & $\mathbf{7 5 \%}$ \\
\hline control & 153.7 & 40.84 & 100 & 228 & 145 & 128 & 181 \\
$1 \%$ & 136.6 & 24.35 & 95 & 166 & 140.5 & 126 & 156 \\
$2 \%$ & 136.8 & 27.14 & 85 & 168 & 143 & 117 & 154 \\
\hline
\end{tabular}

etp

\begin{tabular}{lccccccc} 
Group & Mean & Std Dev & Min & Max & Med & $\mathbf{2 5 \%}$ & $\mathbf{7 5 \%}$ \\
\hline control & 199.3 & 33.92 & 150 & 265 & 194 & 175 & 218 \\
$1 \%$ & 146.8 & 23.54 & 111 & 180 & 142.5 & 135 & 161 \\
$2 \%$ & 149.7 & 24.51 & 112 & 194 & 150 & 138 & 161 \\
\hline
\end{tabular}

5 min

\begin{tabular}{lccccccc} 
Group & Mean & Std Dev & Min & Max & Med & $\mathbf{2 5 \%}$ & $\mathbf{7 5 \%}$ \\
\hline control & 132.2 & 29.94 & 87 & 168 & 131 & 108 & 162 \\
$1 \%$ & 116.7 & 25.61 & 80 & 153 & 122 & 88 & 136 \\
$2 \%$ & 121.0 & 30.43 & 89 & 195 & 110 & 106 & 125 \\
\hline
\end{tabular}

$10 \min$

\begin{tabular}{lccccccc} 
Group & Mean & Std Dev & Min & Max & Med & $\mathbf{2 5 \%}$ & $\mathbf{7 5 \%}$ \\
\hline control & 115.9 & 21.26 & 91 & 155 & 115 & 98 & 129 \\
$1 \%$ & 114.4 & 23.86 & 83 & 151 & 118 & 88 & 131 \\
$2 \%$ & 116.0 & 22.40 & 90 & 166 & 114 & 99 & 122 \\
\hline
\end{tabular}


c. Arterial plasma concentration of adrenaline $\left(\mathrm{pcg} \mathrm{ml^{-1 }}\right)$.

baseline

\begin{tabular}{lccccccc} 
Group & Mean & Std Dev & Min & Max & Med & $\mathbf{2 5 \%}$ & $\mathbf{7 5 \%}$ \\
\hline Control & 124.15 & 72.6 & 40.83 & 255.16 & 126.16 & 45.81 & 171.58 \\
$1 \%$ & 152.87 & 85.44 & 48.10 & 329.61 & 156.85 & 82.76 & 202.04 \\
$\mathbf{2 \%}$ & 55.19 & 27.94 & 14.22 & 115.20 & 51.39 & 44.54 & 65.04 \\
\hline
\end{tabular}

drug

\begin{tabular}{lccccccc} 
Group & Mean & Std Dev & Min & Max & Med & $\mathbf{2 5 \%}$ & $\mathbf{7 5 \%}$ \\
\hline control & 78.84 & 80.84 & 22.82 & 247.93 & 35.69 & 26.18 & 142.0 \\
$1 \%$ & 66.92 & 80.96 & 19.25 & 206.92 & 45.98 & 34.59 & 127.05 \\
$2 \%$ & 39.18 & 16.74 & 14.08 & 75.32 & 38.45 & 31.05 & 45.47 \\
\hline
\end{tabular}

etp

\begin{tabular}{lccccccc} 
Group & Mean & Std Dev & Min & Max & Med & $\mathbf{2 5 \%}$ & $\mathbf{7 5 \%}$ \\
\hline control & 180.27 & 181.1 & 40.99 & 127.48 & 127.48 & 65.41 & 216.14 \\
$1 \%$ & 66.92 & 80.96 & 17.31 & 267.78 & 33.10 & 21.25 & 66.94 \\
$2 \%$ & 45.51 & 28.68 & 11.35 & 110.25 & 38.05 & 28.15 & 45.98 \\
\hline
\end{tabular}

5 min

\begin{tabular}{lccccccc} 
Group & Mean & Std Dev & Min & Max & Med & $\mathbf{2 5 \%}$ & $\mathbf{7 5 \%}$ \\
\hline control & 81.0 & 83.36 & 25.0 & 246.29 & 34.59 & 31.17 & 147.47 \\
$1 \%$ & 57.01 & 57.71 & 16.51 & 180.23 & 37.54 & 19.34 & 52.55 \\
$2 \%$ & 50.74 & 51.33 & 7.7 & 160.38 & 34.14 & 15.85 & 59.67 \\
\hline
\end{tabular}

$10 \min$

\begin{tabular}{lccccccc} 
Group & Mean & Std Dev & Min & Max & Med & $\mathbf{2 5 \%}$ & $\mathbf{7 5 \%}$ \\
\hline control & 76.95 & 61.59 & 17.84 & 188.1 & 58.84 & 22.75 & 133.46 \\
$1 \%$ & 60.76 & 75.69 & 14.80 & 220.80 & 25.24 & 20.04 & 42.78 \\
$\mathbf{2 \%}$ & 72.37 & 120.40 & 10.07 & 388.76 & 32.49 & 14.59 & 57.57 \\
\hline
\end{tabular}

d. Arterial plasma concentration of noradrenaline ( $\left.\mathrm{pcg} \mathrm{ml}{ }^{-1}\right)$.

baseline

\begin{tabular}{lccccccc} 
Group & Mean & Std Dev & Min & Max & Med & $\mathbf{2 5 \%}$ & $\mathbf{7 5 \%}$ \\
\hline control & 321.94 & 177.12 & 140.81 & 629.60 & 262.41 & 188.74 & 451.43 \\
$1 \%$ & 306.67 & 151.38 & 126.97 & 593.29 & 277.76 & 205.15 & 395.49 \\
$2 \%$ & 288.98 & 111.55 & 177.77 & 481.11 & 245.17 & 205.31 & 376.01 \\
\hline
\end{tabular}

drug

\begin{tabular}{lccccccc} 
Group & Mean & Std Dev & Min & Max & Med & $\mathbf{2 5 \%}$ & $\mathbf{7 5 \%}$ \\
\hline control & 284.57 & 132.47 & 161.68 & 563.97 & 228.12 & 203.17 & 335.56 \\
$1 \%$ & 246.26 & 66.03 & 183.59 & 396.69 & 222.82 & 202.29 & 265.28 \\
$\mathbf{2 \%}$ & 295.44 & 145.83 & 102.94 & 577.31 & 275.96 & 210.36 & 349.77 \\
\hline
\end{tabular}

etp

\begin{tabular}{lccccccc} 
Group & Mean & Std Dev & Min & Max & Med & $\mathbf{2 5 \%}$ & $\mathbf{7 5 \%}$ \\
\hline control & 373.99 & 165.85 & 203.44 & 676.56 & 325.79 & 230.13 & 496.13 \\
$1 \%$ & 239.65 & 87.90 & 149.98 & 416.77 & 204.73 & 184.70 & 249.98 \\
$\mathbf{2 \%}$ & 264.89 & 105.38 & 89.43 & 452.27 & 255.85 & 201.20 & 334.02 \\
\hline
\end{tabular}

5 min

\begin{tabular}{lccccccc} 
Group & Mean & Std Dev & Min & Max & Med & $\mathbf{2 5 \%}$ & $\mathbf{7 5 \%}$ \\
\hline control & 331.85 & 149.95 & 218.19 & 684.33 & 265.10 & 232.25 & 392.60 \\
$1 \%$ & 267.72 & 121.56 & 101.10 & 513.45 & 230.79 & 210.41 & 292.10 \\
$\mathbf{2 \%}$ & 278.89 & 102.54 & 94.15 & 406.79 & 327.31 & 207.75 & 354.01 \\
\hline
\end{tabular}

\begin{tabular}{lccccccc}
$\mathbf{1 0}$ min & & & & & & & \\
Group & Mean & Std Dev & Min & Max & Med & 25\% & $\mathbf{7 5 \%}$ \\
\hline control & 346.22 & 205.13 & 172.2 & 780.17 & 241.90 & 230.13 & 443.18 \\
$1 \%$ & 296.57 & 171.19 & 120.77 & 661.26 & 244.86 & 175.11 & 410.09 \\
$2 \%$ & 273.38 & 96.69 & 138.23 & 373.67 & 288.01 & 193.69 & 364.10 \\
\hline
\end{tabular}




\section{Appendix E.}

EndobronchialDrugandGasApplicationduringReanimation Tube (EDGAR-Tube)

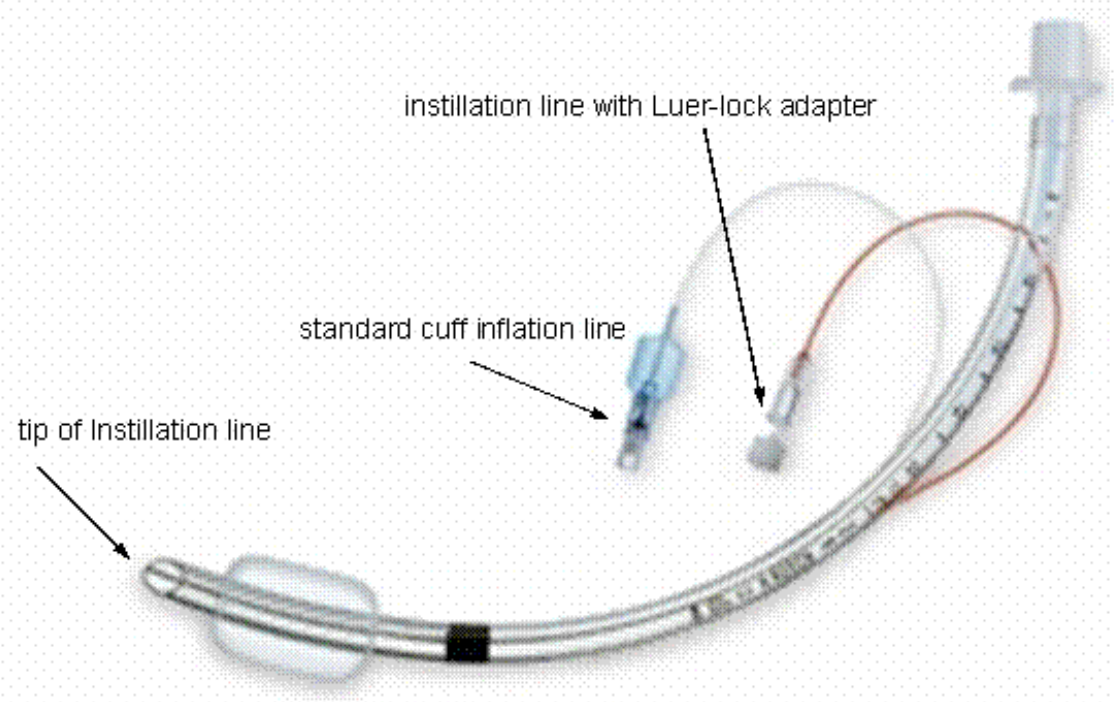

Dr. Florian Heid@

\section{Appendix F}

Trachlight $\odot$, device and usage

powerful lightsource
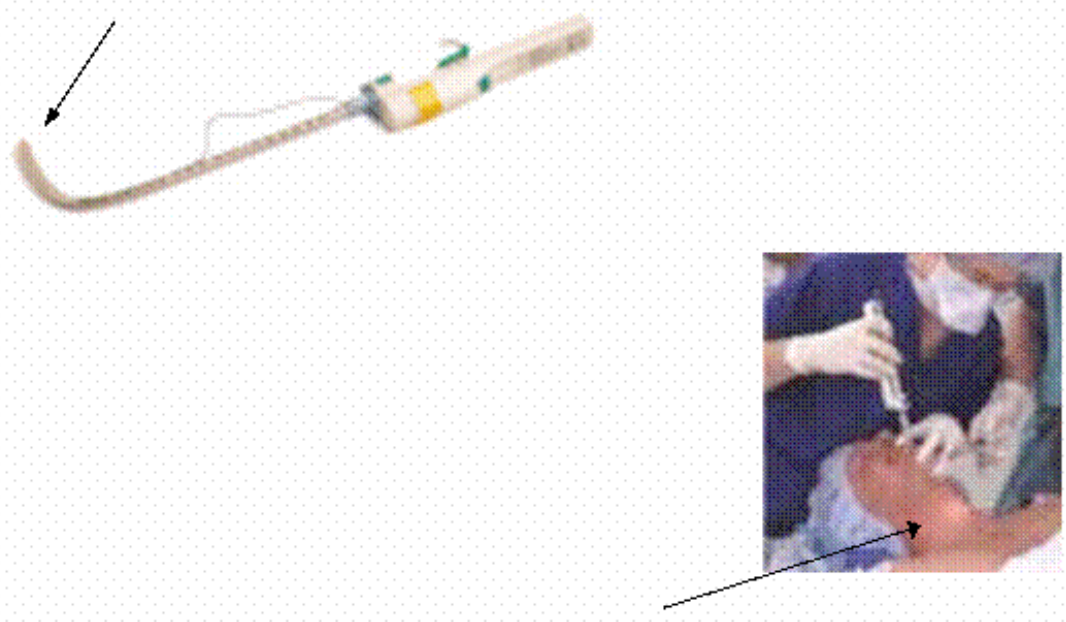

Transcutaneous glow for anatomic orientation and guidance 


\section{REFERENCES}

[1] Prys-Roberts C, Greene LT, Meloche R, Foex P. Studies of anaesthesia in relation to hypertension: haemodynamic consequences of induction and endotracheal intubation. Br J Anaesth 1971; 43: 53147.

[2] Low JM, Harvey JT, Prys-Roberts C, Dagnino J. Studies of anaesthesia in relation to hypertension. VII: Adrenergic responses to laryngoscopy. Br J Anaesth 1986; 58: 471-77.

[3] Takahashi S, Mizutani T, Miyabe M. Hemodynamic responses to tracheal intubation with laryngoscope versus lightwand device (trachlight) in adults with normal airway. Anesth Analg 2002; 95: 480-84.

[4] Nishikawa K, Omote K, Kawana S, Namiki A. A comparison of hemodynamic changes after endotracheal intubation using the lightwand device and the laryngoscope in normotensive and hypertensive patients. Anesth Analg 2000; 90: 1203-07.

[5] Hirabayashi Y, Hiruta M, Kawakami T, et al. Effects of lightwand (Trachlight) compared with direct laryngoscopy on circulatory responses to tracheal intubation. Br J Anaesth 1998; 81: 253-55.

[6] Kihara S, Brimacombe J, Yaguchi Y, Watanabe S, Taguchi N, Komatsuzaki T. Hemodynamic responses among three tracheal intubation devices in normotensive and hypertensive patients. Anesth Analg 2003; 96: 890-05.

[7] Kanaide M, Fukusaki M, Tamuea S, Takada M, Miyakado M, Sumikawa K. Hemodynamic and catecholamine responses during tracheal intubation using a lightwand device (Trachlight) in elderly patients with hypertension. J Anesth 2003; 17: 161-65.

[8] Derbyshire DR, Smith G, Achola KJ. Effect of topical lidocaine on the sympathoadrenal responses to tracheal intubation. Br J Anaesth 1987; 59: 300-04.

[9] Denlinger KJ, Ellison N, Ominsky AJ. Effects of intratracheal lidocaine on circulatory responses to tracheal intubation. Anesthesiology 1974; 41: 409-12.

[10] Stoelting RK. Circulatory changes during direct laryngoscopy and tracheal intubation. Anesthesiology 1977; 47: 381-83.
[11] Russel WJ, Morris RG, Frewin DB, Drew SE. Changes in plasma catecholamine concentrations during endotracheal intubation. $\mathrm{Br} \mathrm{J}$ Anaesth 1981; 53: 837-39.

[12] Hipp R, Mielke L, Hargasser S, Entholner E, Niggeschmidt B, von Hundelshausen B. A new tube for endobronchial application of drugs. Fortschr Med 1990; 108: 550-53.

[13] Cohen J, Ed. Statistical Power Analysis for the Behavioural Sciences. Lawrence Erlbaum Associates, Hillsdale, $2^{\text {nd }}$ ed. New Jersey, 1988.

[14] Kayhan Z, Aldemir D, Mutlu H, Ögüs E. Which is responsible for the hemodynamic response due to laryngoscopy and endotrachea intubation? Catecholamines, vasopressin or angiotensin? Eur J Anaesth 2005; 22: 780-85.

[15] Pernerstorfer T, Krafft P, Fitzgerald RD, et al. Stress response to tracheal intubation: direct laryngoscopy compared with blind oral intubation. Anaesthesia 1995; 50: 17-22

[16] Stoelting RK. Sympathomimetics. In: Stoelting RK, Eds. Pharmacology \& Physiology in Anesthetic Practise. $3^{\text {rd }}$ ed. Philadelphia New York: Lippincott-Raven 1999; 259-77.

[17] Kovac AL. Controlling the hemodynamic response to laryngoscopy and endotracheal intubation. J Clin Anesth 1996; 8: 63-79.

[18] Ugur B, Ogurlu M, Gezer E, Nuri Aydin O, Gürsoy F. Effects of esmolol, lidocaine and fentanyl on haemodynamic responses to endotracheal intubation: a comparative study. Clin Drug Investig 2007; 27: 269-77.

[19] Rosenberg MB, Levesque PR, Bourke DL. Use of the LTA kit as a guide for endotracheal intubation. Anesth Analg 1977; 56: 287-88.

[20] Williams KA, Barker GL, Harwood RJ, Woodall NM. Combined nebulization and spray-as-you-go topical local anaesthesia of the airway. Br J Anaesth 2005; 95: 549-53.

[21] Catterall WA, Mackie K. Local Anesthestics. In: Brunton LL, Lazo JS, Parker K, Eds. Goodman and Gilman's, The Pharmacological Basis of Therapeutics, $11^{\text {th }}$ ed. New York: McGraw-Hill, 2005; 369-87.

(C) Dünges et al.; Licensee Bentham Open.

This is an open access article distributed under the terms of the Creative Commons Attribution License (http: //creativecommons.org/licenses/by/2.5/), which permits unrestrictive use, distribution, and reproduction in any medium, provided the original work is properly cited. 